\title{
Tribotronic bipolar junction transistor for mechanical frequency monitoring and use as touch switch
}

\author{
Fengben $\mathrm{Xi}^{1,2}$, Yaokun Pang ${ }^{1,2}$, Wenjian $\mathrm{Li}^{1,2}$, Tianzhao Bu ${ }^{1,2}$, Junqing Zhao ${ }^{1,2}$, Guoxu Liu ${ }^{1,2}$, Tong Guo ${ }^{1}$,
} Wenbo Liu ${ }^{1}$ and Chi Zhang $\mathbb{B}^{1,2}$

\begin{abstract}
Tribotronics, a new field that involves the coupling of triboelectricity and semiconductors, has attracted great interest in the nanoenergy and nanoelectronics domains. This paper proposes a tribotronic bipolar junction transistor (TBJT) that incorporates a bipolar junction transistor and a triboelectric nanogenerator (TENG) in the single-electrode mode. When the mobile triboelectric layer slides on the device surface for electrification, a bias voltage is created and applied to the emitter junction, and then the base current from the TENG is amplified. Based on the fabricated TBJT, a mechanical frequency monitoring sensor with high sensitivity and excellent stability and a finger-triggered touch switch were developed. This work demonstrated for the first time a tribotronic device with simultaneously controlled voltage and current voltage/current simultaneously controlled tribotronic device, which has promising potential applications in micro/nano-sensors, human-machine interactions, intelligent instrumentation, wearable electronics, and other applications.
\end{abstract}

\section{Introduction}

In the last few decades, transistor scaling has followed the well-known Moore's law, resulting in an increase of two times in the transistor density every 2 years ${ }^{1,2}$, which is approaching the limitations of physical size and power $^{3,4}$. Therefore, information technology (IT) in the post-Moore's law period will develop toward new directions including diversification, improved sensors, multifunctionality, and self-powering capacities ${ }^{5-10}$. As one of the basic transistors, the bipolar junction transistor (BJT) still plays an important role in the design of discrete and very-high-frequency circuits and is used as an amplifier or switch because of its high transconductance and output resistance compared to MOSFETs ${ }^{11,12}$. However, the

\footnotetext{
Correspondence: Chi Zhang (czhang@binn.cas.cn)

${ }^{1}$ CAS Center for Excellence in Nanoscience, Beijing Key Laboratory of Micronano Energy and Sensor, Beijing Institute of Nanoenergy and Nanosystems, Chinese Academy of Sciences, 100083 Beijing, China

${ }^{2}$ School of Nanoscience and Technology, University of Chinese Academy of Sciences, 100049 Beijing, China

These authors contributed equally: Fengben Xi, Yaokun Pang
}

traditional BJT is modulated by internal electrical signals and lacks the direct interaction mechanism between the external environment and electronics.

Recently, the invention of a triboelectric nanogenerator (TENG) has successfully provided an effective approach to convert ambient mechanical energy into electricity ${ }^{13}$. The working principle of the TENG is based on contact electrification and electrostatic induction, which have been widely used in micro/nano-energy ${ }^{14-17}$, selfpowered systems ${ }^{18-26}$, and blue energy ${ }^{27-29}$. Furthermore, by coupling the triboelectricity with semiconductors, a new field of tribotronics has been proposed $^{30-32}$, which concerns research regarding the interaction between triboelectricity and semiconductors ${ }^{33}$ using triboelectric potential controlling electrical transport and transformation in semiconductors for information sensing and active control ${ }^{34-36}$. To date, various tribotronic devices have been developed including contact-gated LEDs ${ }^{37}$, touch memory ${ }^{38}$, adjustable phototransistors $^{39,40}$, sensing arrays ${ }^{41}$, tribotronic tuning 

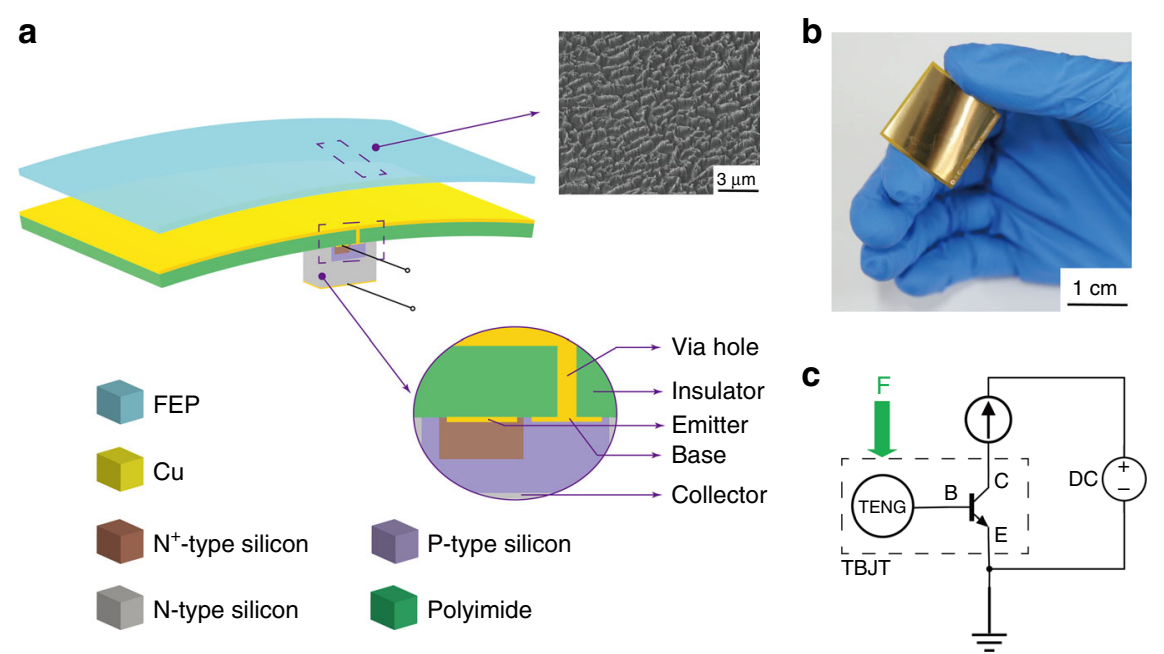

Fig. 1 Basic structure of the tribotronic bipolar junction transistor (TBJT). a Schematic illustration of the TBJT. Inset: cross-section configuration of the TBJT, showing an electrical connection between the TENG in the single-electrode mode and an n-p-n bipolar junction by via hole; SEM image of nanostructures on the surface of the mobile triboelectric layer made of FEP film. $\mathbf{b}$ Optical photograph of the TBJT. $\mathbf{c}$ Equivalent circuit of the TBJT

diodes $^{42}$, and flexible organic tribotronic transistor ${ }^{43}$. All these applications demonstrate the potential of tribotronics in active interaction electronics, offering a prospective strategy to design smart sensors with the advantages of low cost, simple mechanism, and excellent sensitivity.

In this study, we developed a tribotronic BJT (TBJT) by combining a triode with a TENG in the single-electrode mode. The collector current of the TBJT can be amplified and tuned by the base current from the TENG with the sliding motion of the mobile triboelectric layer. Based on the fabricated TBJT, a mechanical frequency monitoring sensor and a finger-triggered active smart touch switch have been developed. In contrast, from previous tribotronic transistors, the TBJT is controlled by the voltage and current created by the TENG simultaneously, which has potential applications in micro/nano-sensors, humanmachine interaction (HMI), intelligent instrumentation, and remote controls.

\section{Results}

\section{Structure of the TBJT}

The basic structure of the TBJT comprises a flexible polyimide substrate, a $\mathrm{Cu}$ pad $(25 \mathrm{~mm} \times 25 \mathrm{~mm})$, a freestanding fluorinated ethylene propylene (FEP) film, and an NPN type triode, as schematically illustrated in Fig. 1a. The $\mathrm{Cu}$ pad is deposited on the top surface of the flexible polyimide substrate, and the triode made of a siliconbased n-p-n junction is constructed on the bottom layer. The emitter, base and collector are electrically connected with the $\mathrm{n}+$-type, the p-type, and the $\mathrm{n}$-type region of the triode, respectively. Through the designed via hole, the $\mathrm{Cu}$ pad is also electrically connected to the base electrode, as shown in the inset of the cross-section. The mobile layer is assembled next to the $\mathrm{Cu}$ pad, which experiences vertical contact and separation from the $\mathrm{Cu}$ pad by the external force. The other inset shows the SEM image of the nanostructures on the surface of the mobile triboelectric layer made of FEP film, which is modified via inductive coupling plasma (ICP) to enhance the surface triboelectric charge density. A well-designed TBJT is presented in Fig. 1b (the detailed fabrication process is introduced in the Methods section). Figure 1c presents the equivalent circuit of the TBJT, which intuitively shows the interaction between the external force and the electronics.

\section{Working mechanism of the TBJT}

The working mechanism of the TBJT is elaborated in Fig. 2, which demonstrates a working principle different from that of the conventional $\mathrm{BJT}$ configuration, based on the coupling of the NPN-BJT, triboelectrification and electrostatic induction. As demonstrated in Fig. 2a, the FEP film is purposely chosen as the mobile triboelectric layer owing to its high electronegativity according to the triboelectric series ${ }^{44}$. The collector of the TBJT is connected with a voltage source, whereas the emitter is grounded.

In the initial position, when the FEP film fully contacts the $\mathrm{Cu}$ pad, equal negative and positive charges are induced on the surfaces of the FEP film and the $\mathrm{Cu}$ pad, respectively, owing to their difference in triboelectric polarity. Because the positive triboelectric charges completely balance out the negative counterpart in this circumstance, there is no electrical potential difference applied to the base region and no charge transfer occurs 


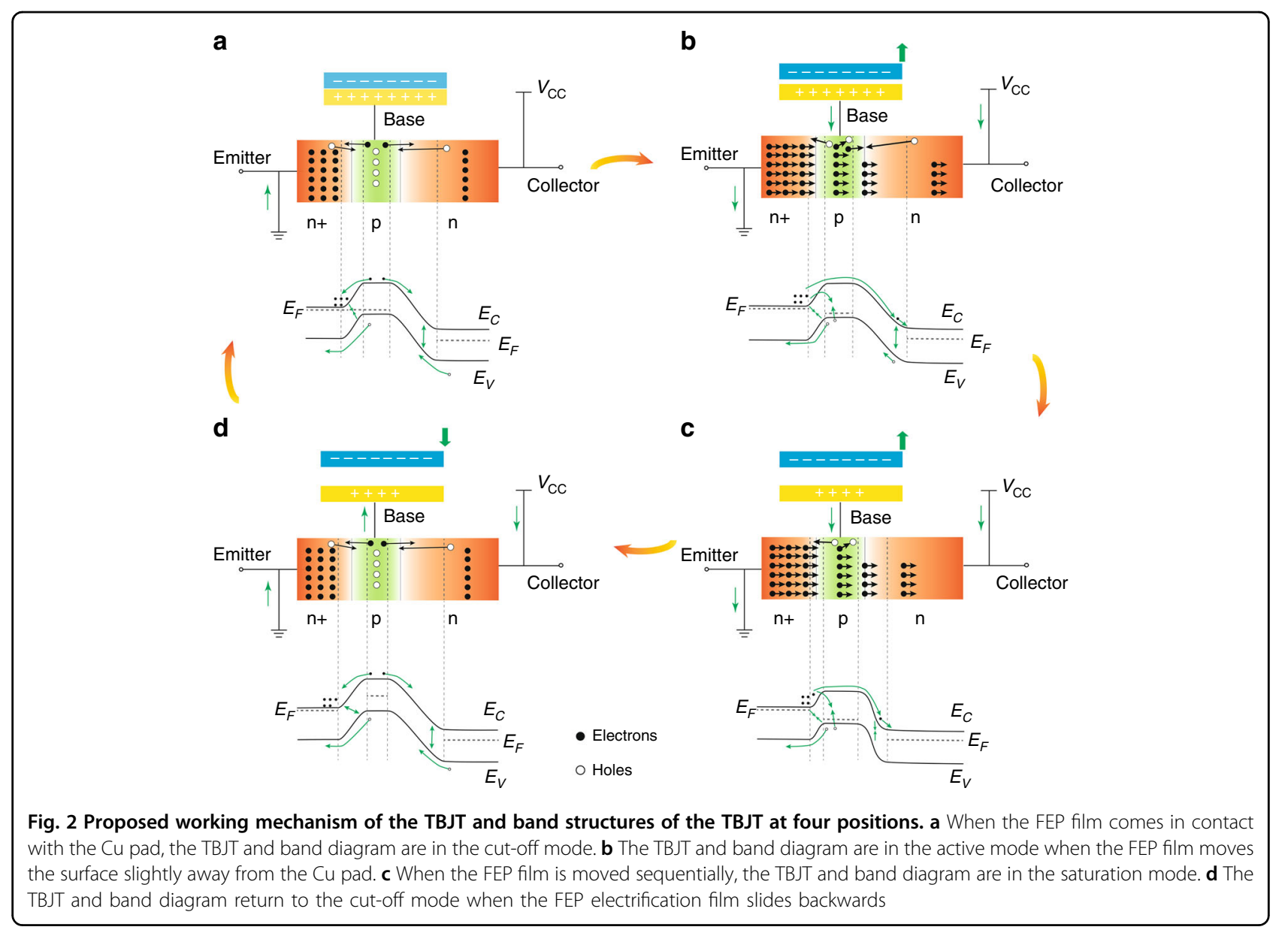

from the TENG. The emitter-base junction is zero offset and the collector-base junction is reverse biased; therefore, the TBJT stays in the cut-off mode, which corresponds to a logical "off" state. Meanwhile, the depletion regions are generated in both the junctions where the electrons and holes recombine. Moreover, the Fermi levels of the base and emitter region in Fig. 2a are horizontally aligned, and are higher than those of the collector in its band diagram.

Once the FEP film is gradually separated from the $\mathrm{Cu}$ surface as the external force is vertically applied, the equilibrium potential is disturbed, resulting in a positive electrical potential being applied to the triode (Fig. 2b). Meanwhile, the emitter-base junction is forward biased (the depletion layer width decreases) and the collector-base junction is reverse biased (the depletion layer width increases); thus, the TBJT shifts into the active mode. In the active mode, the electrons are injected from the forward biased $\mathrm{n}+$-type emitter region into the $\mathrm{p}$ type base where they diffuse as minority carriers to the reverse-biased $n$-type collector and are swept away by the electric field in the reverse-biased collector-base junction. Then, based on the law of charge conservation, the current from the TENG is amplified dramatically in the collector by the triode. The Fermi level of the base region in Fig. $2 \mathrm{~b}$ decreases owing to the positive potential in the base. Next, as shown in Fig. 2c, when the movement of the FEP film continues, the inner electric field gradually increases as well. Therefore, with both junctions being forward-biased (widths of both depletion layers decrease), the TBJT enters the saturation mode, and the Fermi level of the base region decreases between the emitter and collector. This mode corresponds to a logical "on," or a closed switch, which means that the collector current has a high magnitude and is stable. Finally, the TBJT and its band diagram return to the cut-off mode when the FEP film slides backwards (Fig. 2d). However, in this process, the Fermi level of the base region increases because of the negative potential in the base.

Figure S1 shows the theoretically calculated results (performed by the software Comsol Multiphysics) of the potential difference generated from the contactseparating and sliding mode TENG with the FEP and copper films, indicating that this triboelectric potential varies with the separation distance of the mobile triboelectric layer. 

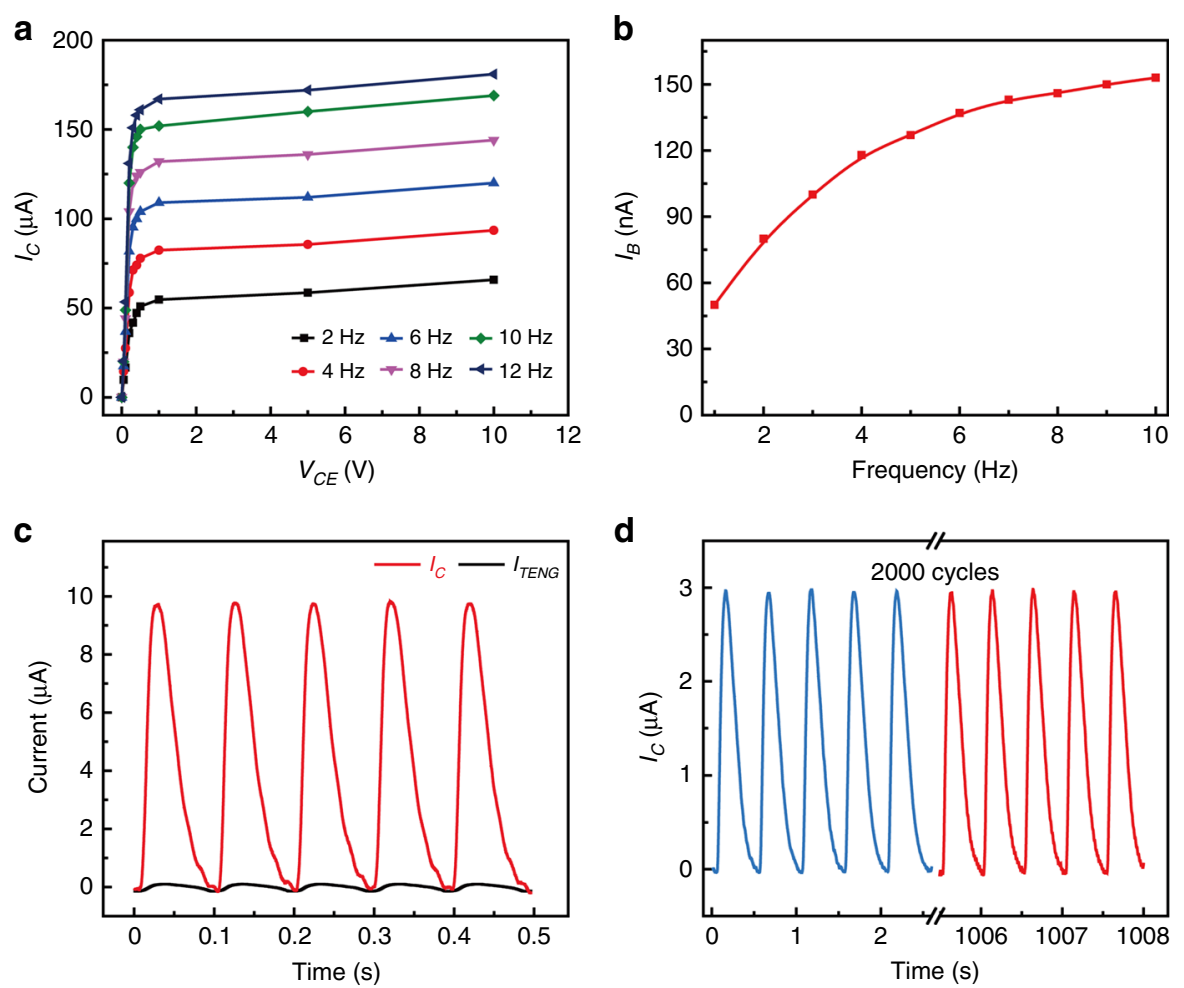

Fig. 3 Electrical characterization of the TBJT. a Output characteristic curves of $V_{C E}-l_{C}$ with different frequencies. b Relationship between the $I_{B}$ and the frequency. $\mathbf{c}$ Amplification curve of collector current and base current at the frequency of $4 \mathrm{~Hz}$ and $V_{C E}=0.5 \mathrm{~V}$. $\mathbf{d}$ Stability test of the sensor. $V_{C E}$ remains at $0.5 \mathrm{~V}$ during the entire experiment. A small hysteresis and good repeatability are attained even after tests of $\sim 2000$ cycles

\section{Electrical characterization of the TBJT}

To better evaluate the performance of the TBJT, its electrical characterizations were systematically studied, as depicted in Fig. 3. The measured results demonstrated satisfactory performance of the BJT. Figure 3a plots the output characteristic of the TBJT, and the separation frequency of the FEP film can be accurately controlled using a linear motor. As shown in the graph, the $I_{\mathrm{C}}$ rises as the separation frequency increases from 2 $\mathrm{Hz}$ to $12 \mathrm{~Hz}$ within a $V_{\mathrm{CE}}$ of $0-10 \mathrm{~V}$. The curves of $I_{\mathrm{B}}$ and frequency were also studied, and are shown in Fig. 3b. When the separation frequency of the FEP film varies from 0 to $10 \mathrm{~Hz}$, the $I_{\mathrm{B}}$ increases from 50 to 153 $\mathrm{nA}$. In addition, the curve of the collector current and base current at the frequency of $4 \mathrm{~Hz}$ and $V_{\mathrm{CE}}=0.5 \mathrm{~V}$ are shown in Fig. 3c, and the amplification factor of the peak current $\left(I_{\mathrm{C}}=9.7469 \mu \mathrm{A}\right.$ and $\left.I_{\mathrm{TENG}}=0.0974 \mu \mathrm{A}\right)$ can be reached at $100 \times$. Moreover, measurements for $\sim 2000$ cycles are carried out to validate the stability and repeatability of the device, as described in Fig. 3d. Even after 2000 test cycles, the changes in the peak collector current $I_{\mathrm{C}}$ are $<5 \%$, showing its small hysteresis and excellent reproducibility. The above experimental results indicate that the collector current increases with the increasing frequency, which corresponds to good electrical characterization. Owing to the direct interaction mechanism between the external environment and electric device, the TBJT is likely to have excellent applications in sensors and HMI.

\section{Discussion}

\section{Application of the TBJT for mechanical frequency monitoring}

First, the TBJT was applied to mechanical frequency monitoring (Fig. 4). Based on the electrical characterization of the TBJT, the $I_{\mathrm{C}}$ can be tuned by the separation frequency of the FEP film. Based on this principle, we have designed a mechanical frequency monitoring sensor based on the TBJT. The structures of the TBJT with contact-separating and sliding single electrode modes, which are developed for vibrational and sliding frequency monitoring, are shown in Fig. 4a, b, respectively. Figure $4 \mathrm{c}-\mathrm{f}$ show the characteristics of the mechanical frequency monitoring sensors. When the FEP film undergoes vertically reciprocating motion with the $\mathrm{Cu}$ film at a frequency from 1 to $5 \mathrm{~Hz}$ with a collector voltage of $0.5 \mathrm{~V}$, the $I_{C}$ increases with the increase in the vibrational frequency, as shown in Fig. 4c. The sensor exhibits good sensing performance with an exceptional sensitivity of $1.00 \mu \mathrm{A} \mathrm{Hz}^{-1}$. Similarly, as demonstrated in Fig. $4 \mathrm{~d}, \mathrm{f}$, 
a

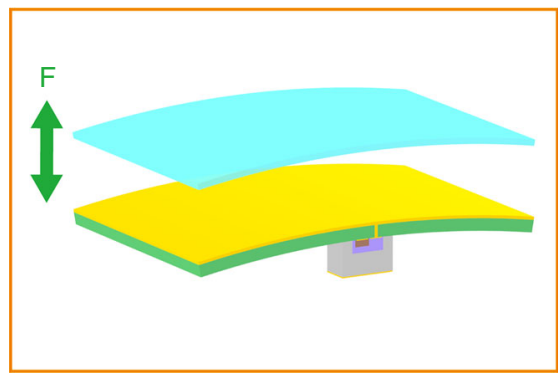

C

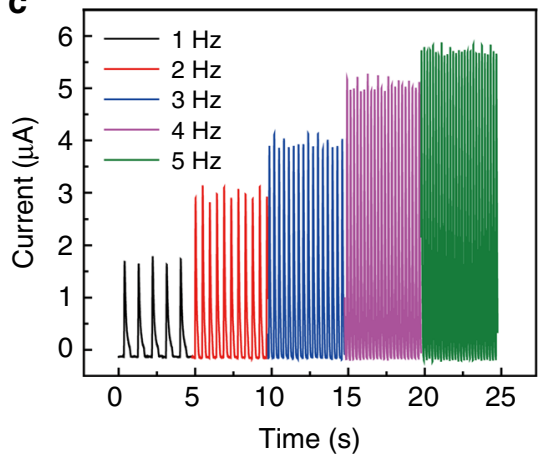

e

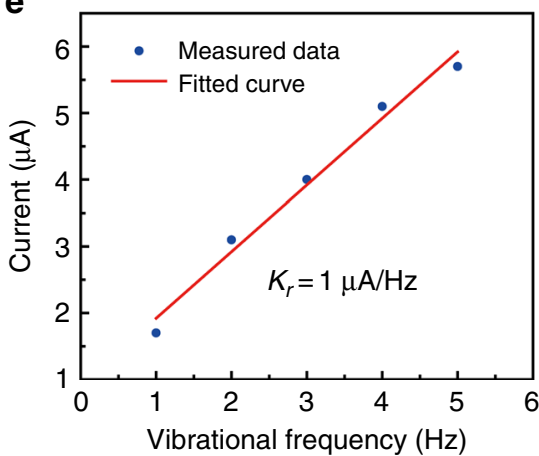

b

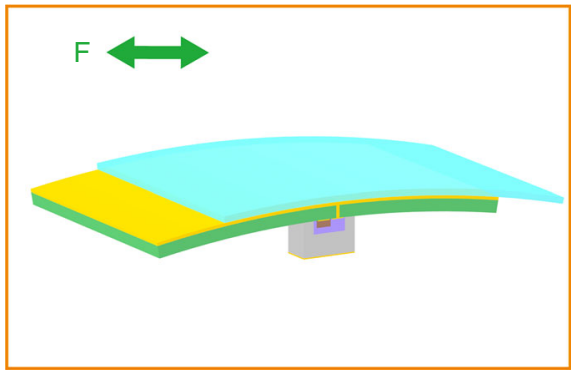

d

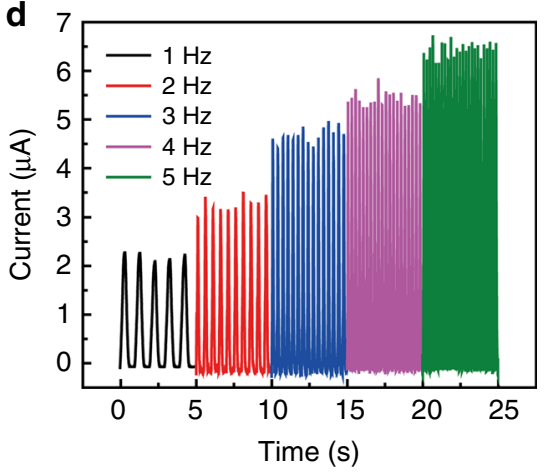

f

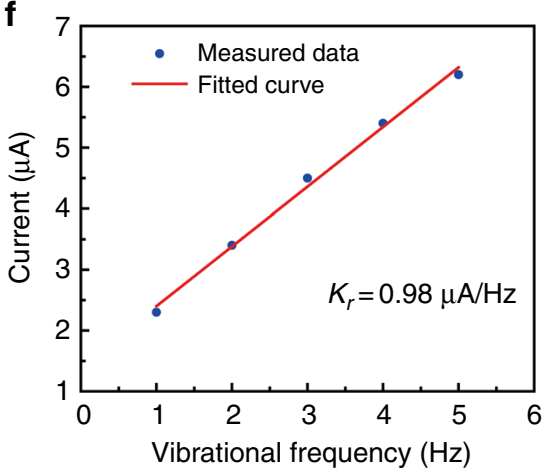

Fig. 4 TBJT for signal frequency monitoring. $\mathbf{a}$, $\mathbf{b}$ Schematic illustration of the device structure with contact-separating mode and sliding mode, respectively. $\mathbf{c}$ The collector current, when the FEP film vertically contacts and separates from the copper film with a frequency ranging between $1-5$ $\mathrm{Hz}$ with collector voltage $0.5 \mathrm{~V}$. d The collector current, when the FEP film and the copper film slide against each other with a frequency ranging between $1-5 \mathrm{~Hz}$ with collector voltage $0.5 \mathrm{~V}$. e Relationship between the collector current and the vibration frequency; the sensitivity is $1.00 \mu \mathrm{A} \mathrm{Hz}{ }^{-1}$. f Relationship between the collector current and sliding frequency; the sensitivity is $0.98 \mu \mathrm{A} \mathrm{Hz}{ }^{-1}$

when the FEP film and the copper film slide against each other, there is a positive correlation between the $I_{\mathrm{C}}$ and the sliding frequency, and the sensitivity is $0.98 \mu \mathrm{A} \mathrm{Hz}^{-1}$. By exploiting the excellent sensitivity, the TBJT as a mechanical frequency monitoring sensor shows promising prospects in HMI and intelligent sensing.

\section{Application of the TBJT as a finger-triggered touch switch in smart home}

In addition to being a mechanical signal sensor, another intriguing application of the TBJT is as a finger-triggered active tactile touch switch for smart home control systems. Traditional transistors cannot be touched by human fingers directly owing to the presence of electrostatic charges. However, on the basis of the strong advantages of the TBJT, we designed a finger-triggered active tactile switch with a high sensitivity that can be used in the fields of HMI and remote control. In this configuration, the human skin (finger) can also act as a mobile triboelectric layer, replacing the FEP film according to the triboelectric series. The equivalent electrical circuits of the TBJT and TENG used for controlling the light-emitting diode (LED) are demonstrated in Fig. 5a. The demo optical graph in the inset shows that an LED is controlled directly by the finger touching or releasing the mobile triboelectric layer (FEP film) of the 
a

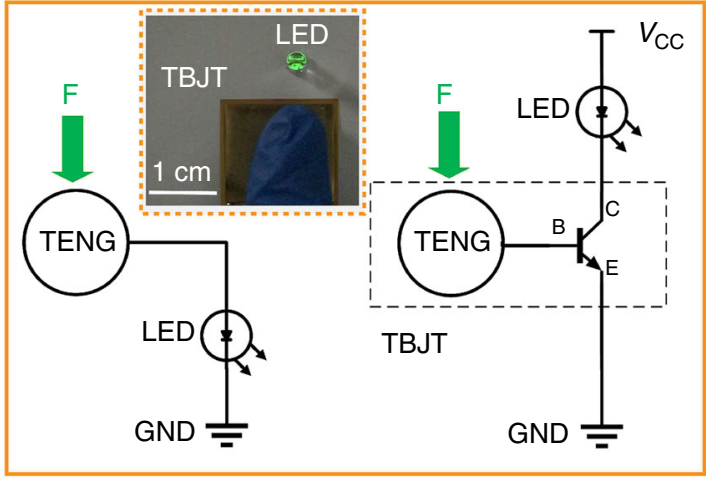

C b

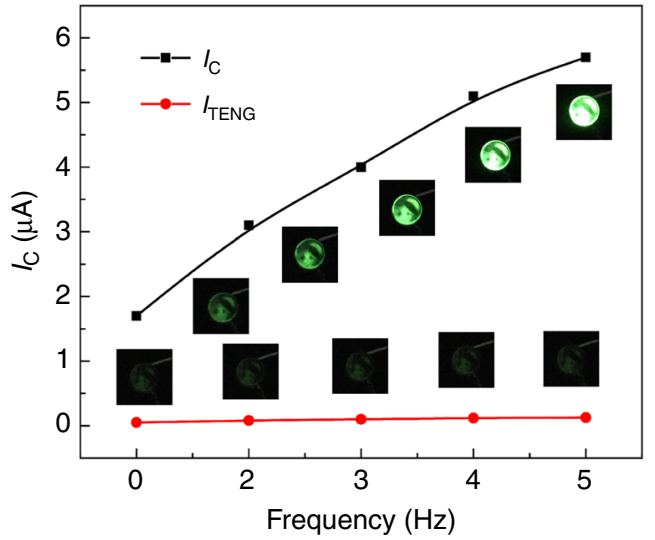

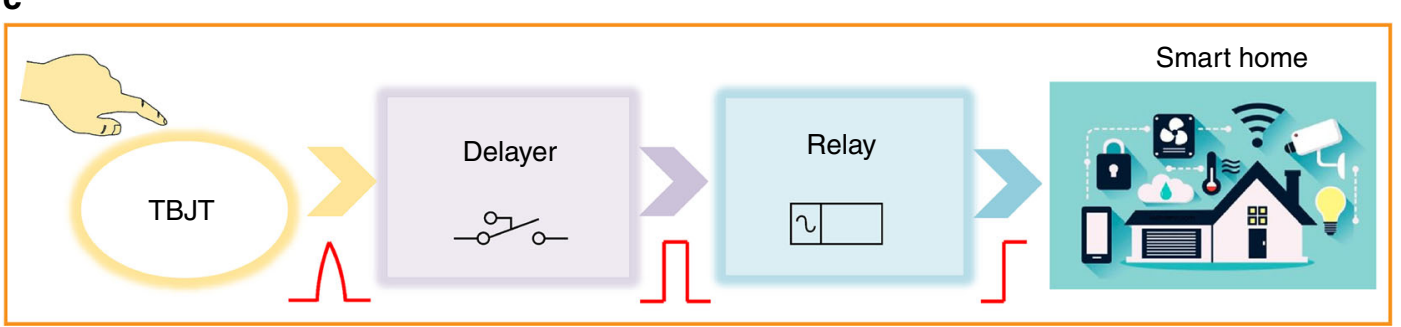

d

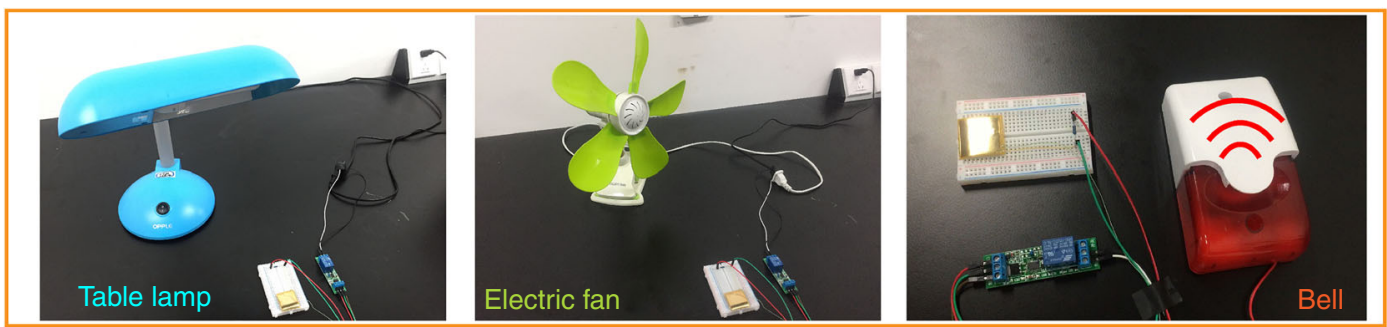

Fig. 5 Application of the TBJT as a finger-triggered touch switch in smart home. a Equivalent electrical circuits of the TBJT and TENG for controlling the LED. Inset showing that an LED can be tuned directly by finger touch or release. $\mathbf{b}$ LED lights are tuned by the external mechanical force at frequencies of $0,1,2,3,4$, and $5 \mathrm{~Hz}$. c Schematic of a TBJT-involved home control system. After the finger touch and release, an impulse signal can be converted into a trigger signal to control the appliances. $\mathbf{d}$ Demonstration of the control of a table lamp, an electric fan, and a household security bell

TBJT. As depicted in Fig. 5b, the LED brightness can be tuned by the switch, and the driving energy of TENG is greatly improved. The LED is turned on/off when the finger presses or releases the switch, and the dynamic demonstrations are shown in Video S1.

Moreover, as shown in Fig. 5c, the smart home control system includes a person; a touch switch based on the TBJT; a simple signal processing circuit; and household appliances, such as a table lamp, an electric fan, and a household security bell. The signal processing module is made up of a delayer and a relay based on a single-chip microcomputer, and the detailed circuit board is demonstrated in Figure S2. When a user touches the $\mathrm{Cu}$ pad of the TBJT, the signal is detected and converted into a switching signal by the signal processing circuit for the household appliances (Fig. 3d and Video S2 to S4).

\section{Materials and methods}

\section{Fabrication of the tribotronic bipolar junction transistor}

First, a $100 \mu \mathrm{m}$ polyimide (PI) substrate $(28 \mathrm{~mm} \times 28$ $\mathrm{mm}$ ) was prepared and cleaned in an ultrasonic cleaner with deionized water, ethanol, and acetone, sequentially, and then it was blow-dried in a drying oven at $100^{\circ} \mathrm{C}$ for $1 \mathrm{~h}$. Subsequently, three localized via holes were drilled on the substrate and $10 \mu \mathrm{m} \mathrm{Cu}$ used as electrical wires were deposited onto the selective area of back side of the substrate according to the designed circuit configuration. Next, layers of $3 \mu \mathrm{m} \mathrm{Ni}$ and $25 \mu \mathrm{m} \mathrm{Cu}$ were electroplated onto the substrate to form the pad $(25 \mathrm{~mm} \times 25 \mathrm{~mm})$. Finally, a chip of the NPN-triode was adhered to the bottom of the PI substrate, in which the base was connected to the $\mathrm{Cu}$ pad through via the hole, and the collector and emitter could be electrically connected to the 
external circuitry by the electrical wires. The mobile layer vertically controlled by the external force was fabricated based on a piece of FEP film, which was also used as a mechanical frequency monitoring sensor.

\section{Surface modification of the FEP film}

First, a $30 \mu \mathrm{m}$ FEP film $(25 \mathrm{~mm} \times 25 \mathrm{~mm})$ was prepared and cleaned. Before etching, a layer of $\mathrm{Cu}$ was RF sputtered onto the surface of the FEP surface, and then $\mathrm{O}_{2}$, Ar, and $\mathrm{CF}_{4}$ gases were fed into the inductively coupled plasma (ICP) chamber to etch the surface with flow rates of 10.0, 15.0, and 30.0 standard cubic centimeter per minute. Then, a $400 \mathrm{~W}$ power source was used to generate the plasma, while another $100 \mathrm{~W}$ power source was used to accelerate the plasma ions moving to the FEP surface. After $5 \mathrm{~min}$, the desired nanostructures were obtained on the $\mathrm{Cu}$ coated FEP surface.

\section{Characterization and electrical measurements of the TBJT}

The current and voltage was quantitatively determined by a linear motor (Akribis-DGL-SH417), a Low Noise Current Preamplifier (SRS-SR570) and a programmable electrometer (Keithley 6514) under ambient conditions at room temperature. A DC power supply (RIGOL-DP-832) was used to supply an electrically applied voltage to the TBJT and the external circuits.

\section{Conclusion}

In summary, we have demonstrated a tribotronic BJT (TBJT) based on a bipolar junction and a TENG in the single-electrode mode. With the sliding of the mobile triboelectric layer, a bias voltage is created, and the base current is amplified. The fabricated TBJT is used as a mechanical frequency monitoring sensor with a high sensitivity $\left(1 \mu \mathrm{A} \mathrm{Hz}^{-1}\right)$ and excellent stability. Moreover, the device can also be used as a finger-triggered active tactile switch. This work has extended emerging tribotronics to a device with simultaneously controlled voltage and current and has demonstrated that the new field may have innovative and promising potential applications in micro/nano-sensors, HMIs, wearable electronics, and other applications.

\section{Acknowledgements}

F.B.X. and Y.K.P. contributed equally to this work. The authors gratefully acknowledge the support of the National Natural Science Foundation of China (No. 51475099), Beijing Talents Foundation (2017000021223TD04), Beijing Nova Program (No. Z171100001117054), the Youth Innovation Promotion Association, CAS (No. 2014033), the "Thousands Talents" program for the pioneer researcher and his innovation team, China, and the National Key Research and Development Program of China (2016YFA0202704).

\section{Conflict of interest}

The authors declare that they have no conflict of interest.

Supplementary Information accompanies this paper at https://doi.org/ 10.1038/s41378-018-0026-1.
Received: 15 April 2018 Revised: 2 June 2018 Accepted: 17 June 2018 Published online: 05 November 2018

References

1. Moore, G. E. Cramming more components onto integrated circuits. Proc. IEEE 86, 82-85 (1998).

2. Moore, G. Progress in digital integrated electronics [Technical literaiture, Copyright 1975 IEEE. Reprinted, with permission. Technical Digest. International Electron Devices Meeting, IEEE, 1975, pp. 11-13.]. IEEE Solid-State Circuits Newsl. 20, 36-37 (2006).

3. Waldrop, M. M. The chips are down for Moore's law. Nature 530, 144-147 (2016).

4. C. Y. Chen et al. 2015 IEEE International Electron Devices Meeting (IEDM). 10.6.1-10.6.4. IEEE, Washington, DC, USA, 2015. https:/doi.org/10.1109/ IEDM.2015.7409671

5. Rogers, J. A., Someya, T. \& Huang, Y. Materials and mechanics for stretchable electronics. Science 327, 1603-1607 (2010).

6. Wang, Z. L. \& Wu, W. Nanotechnology-enabled energy harvesting for selfpowered micro-/nanosystems. Angew. Chem. - Int. Ed. 51, 11700-11721 (2012).

7. Wang, Z. L. Progress in piezotronics and piezo-phototronics. Adv. Mater. 24 4632-4646 (2012).

8. Wang, Z. L. Triboelectric nanogenerators as new energy technology for selfpowered systems and as active mechanical and chemical sensors. ACS Nano 7, 9533-9557 (2013)

9. Crone, B. et al. Large-scale complementary integrated circuits based on organic transistors. Nature 403, 521-523 (2000).

10. $\mathrm{Wu}, \mathrm{H}_{\text {., Huang, } Y .,}$ Xu, F., Duan, Y. \& Yin, Z. Energy harvesters for wearable and stretchable electronics: from flexibility to stretchability. Adv. Mater. 28, 9881-9919 (2016).

11. Bauer, F. D. The super junction bipolar transistor: a new silicon power device concept for ultra low loss switching applications at medium to high voltages. Solid. State Electron. 48, 705-714 (2004).

12. Larson, L. E. Integrated circuit technology options for RFIC's-present status and future directions. in. Custom Integrated Circuits Conference, 1997., Proceedings of the IEEE 1997, 169-176 (1997).

13. Fan, F. R., Tian, Z. Q. \& Lin Wang, Z. Flexible triboelectric generator. Nano Energy 1, 328-334 (2012)

14. Zhou, T. et al. Multilayered electret films based triboelectric nanogenerator. Nano Res. 9, 1442-1451 (2016).

15. Zhou, T. et al. Woven structured triboelectric nanogenerator for wearable devices. ACS Appl. Mater. Interfaces 6, 14695-14701 (2014).

16. Zhang, C., Tang, W., Han, C., Fan, F. \& Wang, Z. L. Theoretical comparison, equivalent transformation, and conjunction operations of electromagnetic induction generator and triboelectric nanogenerator for harvesting mechanical energy. Adv. Mater. 26, 3580-3591 (2014).

17. Zhang, C. et al. Rotating-disk-based direct-current triboelectric nanogenerator. Adv. Energy Mater. 4, 1-7 (2014).

18. Wang, $X$. et al. Self-powered high-resolution and pressure-sensitive triboelectric sensor matrix for real-time tactile mapping. Adv. Mater. 28, 2896-2903 (2016).

19. Pang, Y. K. et al. Triboelectric nanogenerators as a self-powered $3 \mathrm{D}$ acceleration sensor. ACS Appl. Mater. Interfaces 7, 19076-19082 (2015).

20. Zhang, L. et al. Transparent paper-based triboelectric nanogenerator as a page mark and anti-theft sensor. Nano Res. 7, 1215-1223 (2014).

21. Bao Han, C. et al. Self-powered velocity and trajectory tracking sensor array made of planar triboelectric nanogenerator pixels. Nano Energy 9, 325-333 (2014).

22. Fu, X. P. et al. Embedded triboelectric active sensors for real-time pneumatic monitoring. ACS Appl. Mater. Interfaces 9, 32352-32358 (2017).

23. Zhang, X.-S. et al. All-in-one self-powered flexible microsystems based on triboelectric nanogenerators. Nano Energy 47, 410-426 (2018).

24. Zhang, X. S. et al. Frequency-multiplication high-output triboelectric nanogenerator for sustainably powering biomedical microsystems. Nano. Lett. 13, 1168-1172 (2013).

25. Tang, Q. et al. Whirligig-inspired triboelectric nanogenerator with ultrahigh specific output as reliable portable instant power supply for personal health monitoring devices. Nano Energy 47, 74-80 (2018).

26. $\mathrm{Pu}, \mathrm{X}$. et al. Eye motion triggered self-powered mechnosensational communication system using triboelectric nanogenerator. Sci. Adv. 3, 1-8 (2017).

27. Wang, Z. L. Catch wave power in floating nets. Nature 542, 159-160 (2017). 
28. Zhang, L. M. et al. Multilayer wavy-structured robust triboelectric nanogenerator for harvesting water wave energy. Nano Energy 22, 87-94 (2016).

29. Feng, L. et al. Hybridized nanogenerator based on honeycomb-like three electrodes for efficient ocean wave energy harvesting. Nano Energy 47, 217-223 (2018).

30. Zhang, C. \& Wang, Z. L. Tribotronics-A new field by coupling triboelectricity and semiconductor. Nano Today 11, 521-536 (2016).

31. Zhang, C., Tang, W., Zhang, L., Han, C. \& Wang, Z. L. Contact electrification field-effect transistor. ACS Nano 8, 8702-8709 (2014).

32. Xi, F. et al. Universal power management strategy for triboelectric nanogenerator. Nano Energy 37, 168-176 (2017).

33. Jiang, $T$. et al. Theoretical study of sliding-electrification-gated tribotronic transistors and logic device. Adv. Electron. Mater. 4, 1700337-1700344 (2018).

34. Peng, W., Yu, R., He, Y. \& Wang, Z. L. Theoretical study of triboelectric-potential gated/driven metal-oxide-semiconductor field-effect transistor. ACS Nano $\mathbf{1 0}$ 4395-4402 (2016).

35. Yang, B. Z., Lin, Y. S. \& Wu, J. M. Flexible contact-electrification field-effect transistor made from the P3HT:PCBM conductive polymer thin film. Appl. Mater. Today 9, 96-103 (2017).
36. Wu, J. M., Lin, Y. H. \& Yang, B. Z. Force-pad made from contact-electrification poly(ethylene oxide)/InSb field-effect transistor. Nano Energy 22, 468-474 (2016).

37. Zhang, C. et al. Organic tribotronic transistor for contact-electrification-gated light-emitting diode. Adv. Funct. Mater. 25, 5625-5632 (2015).

38. Li, J. et al. Flexible organic tribotronic transistor memory for a visible and wearable touch monitoring system. Adv. Mater. 28, 106-110 (2016).

39. Pang, Y. et al. Tribotronic enhanced photoresponsivity of a MOS2phototransistor. Adv. Sci. 3, 1500419-1500425 (2015).

40. Zhang, C. et al. Tribotronic phototransistor for enhanced photodetection and hybrid energy harvesting. Adv. Funct. Mater. 26, 2554-2560 (2016).

41. Yang, Z. W.et al. Tribotronic transistor array as an active tactile sensing syste $\mathrm{m}$. ACS Nano 10, 10912-10920 (2016).

42. Zhou, T. et al. Tribotronic tuning diode for active analog signal modulation. ACS Nano 11, 882-888 (2017).

43. Zhao, J. et al. Flexible organic tribotronic transistor for pressure and magnetic sensing. ACS Nano 11, 11566-11573 (2017).

44. Davies, D. K. Charge generation on dielectric surfaces. J. Phys. D Appl. Phys. 2, 1533-1537 (1969). 